Z Rheumatol 2006 $65: 217-224$

DOI 10.1007/s00393-006-0052-5

Online publiziert: 3. Mai 2006

(c) Springer Medizin Verlag 2006
M. Østensen

Klinik für Rheumatologie und Klinische Immunologie/Allergologie,

Inselspital Bern, Universitätsklinik, Bern

\title{
Antirheumatische Therapie und Reproduktion
}

\author{
Einfluss auf Fertilität, \\ Schwangerschaft und Stillzeit
}

Eine antirheumatische oder immunsuppressive Therapie bei Frauen und Männern in fertilem Alter kann sich negativ auf die Reproduktion auswirken. Mehrere Medikamente beeinträchtigen die Fertilität, vorübergehend oder permanent. Eine reversible Infertilität kann bei Männern unter Behandlung mit Sulfasalazin auftreten, bei Frauen unter Behandlung mit nichtsteroidalen Antirheumatika (NSAR). Irreversible Infertilität wird häufig durch alkylierende Zytostatika wie Cyclophosphamid verursacht.

Bei vorliegendem Kinderwunsch und bei Bedarf einer kontinuierlichen antirheumatischen Therapie stellt sich die Frage, ob die Therapie mit einer Schwangerschaft vereinbar ist. Einerseits stellen $\mathrm{Ne}$ benwirkungen der Medikamente ein Risiko für die Mutter und den Fetus dar, andererseits kann das Absetzen der Therapie zu einer Verschlechterung der Krankheit führen. Die Informationen in den Arzneimittellisten sind bei der Entscheidung für oder gegen das Fortführen einer Therapie oft wenig hilfreich. Bei neuen Medikamenten werden niemals kontrollierte Studien an Schwangeren oder Stillenden durchgeführt. Die Verträglichkeit von Medikamenten während der Schwangerschaft und Stillzeit stützt sich daher auf experimentelle Daten und Tierversuche, die oft mit sehr hohen, unphysiologischen Dosen einer Substanz durchgeführt werden.

Im Laufe der Zeit erscheinen Berichte, die sich mit einer nicht beabsichtigten Exposition von Schwangeren befassen. Sol- che Daten akkumulieren langsam und teilen in Form von Fallberichten bevorzugt negative Erfahrungen mit. Die Hersteller von Medikamenten sind nicht verpflichtet, ihre Arzneimittelinformationen laufend zu aktualisieren. So kann es vorkommen, dass bei einer Substanz, die keine fruchtschädigende Wirkung zeigt, dies nicht in der Arzneimittelliste aufgeführt wird.

\section{( Für neue Medikamente fehlen Studien an Schwangeren und Stillenden}

Die Tatsache, dass bei so vielen Medikamenten eine Warnung im Hinblick auf Schwangerschaft und Stillzeit ausgesprochen wird, beruht nicht darauf, dass es so viele toxische Medikamente gibt, sondern dass ihr Einfluss auf die humane Schwangerschaft und Stillzeit nicht oder unzureichend untersucht ist.

Die Aktivität der rheumatischen Krankheit ist ausschlaggebend für die Entscheidung ob, wann und wie lange eine medikamentöse Behandlung während der Schwangerschaft nötig ist. Der Verlauf der verschiedenen rheumatischen Krankheiten während der Schwangerschaft ist unterschiedlich. Polyartikuläre Arthritiden wie die rheumatoide Arthritis (RA) und die juvenile idiopathische Arthritis (JIA) bessern sich bei einem Großteil der Patientinnen. Dagegen können die ankylosierende Spondylitis (AS) und die Kollagenosen wie der systemische Lupus erythematodes (SLE) aktiv sein oder sich auch während einer Schwangerschaft verschlechtern. Der Bedarf für eine medikamentöse Behandlung nimmt also bei einigen Patientinnen während der Schwangerschaft ab, bei anderen jedoch $\mathrm{zu}$.

Bei einem aktiven Krankheitsverlauf muss man entscheiden, welche Medikamente ohne Schaden für Mutter und Kind angewandt werden können. Die nachfolgende Übersicht fasst Berichte der Literatur im Hinblick auf antirheumatische Medikamente und Reproduktion zusammen [22].

\section{Medikamente und Fertilität}

\section{Reversible Infertilität durch NSAR}

Mehrere Fallberichte haben eine reversible Infertilität unter der Behandlung mit Indomethacin, Diclofenac, Piroxicam und Naproxen beobachtet [15]. Untersuchungen an Tieren und am Menschen haben gezeigt, dass Hemmer der Cyklooxygenase 1 und 2 den Eisprung verzögern oder verhindern können. Leider ist nicht klar, wie häufig NSAR eine Hemmung des Eisprungs auslösen, bei welcher Dosis und für wie lange die Wirkung andauert. Die meisten Frauen mit rheumatischen Krankheiten, die mit NSAR behandelt werden, sind nicht infertil. Wesentlich ist bei Patientinnen, die trotz Kinderwunsch nicht schwanger werden, die Möglichkeit eines durch 
Tab. 1 Antirheumatika und Fertilität

\begin{tabular}{|c|c|c|}
\hline Medikament & Beeinträchtigung der Fertilität & Kommentare \\
\hline NSAR & Können die Ovulation hemmen & $\begin{array}{l}\text { Häufigkeit der Ovulationshem- } \\
\text { mung unbekannt }\end{array}$ \\
\hline Sulfasalazin & \& nein, $\sigma^{*} \mathrm{ja}$ & $\begin{array}{l}\text { Oligospermie ist reversibel } \\
\text { 2-3 Monate nach Absetzen der } \\
\text { Therapie }\end{array}$ \\
\hline $\begin{array}{l}\text { Chloroquin und Hydroxy- } \\
\text { chloroquin }\end{array}$ & Nein & Keine \\
\hline Cyclosporin A & Nein & Keine \\
\hline Azathioprin & Nein & Keine \\
\hline Methotrexat (MTX) & \$ nein, $\sigma^{\nearrow}$ Oligospermie selten & $\begin{array}{l}\text { Nicht bekannt ob mutagen, daher } \\
\text { auch bei } \sigma^{\star} \text { Absetzen } 3 \text { Monate vor } \\
\text { geplanter Schwangerschaft }\end{array}$ \\
\hline Cyclophosphamid & Gonadotoxisch bei 9 und $\sigma^{\top}$ & $\begin{array}{l}\text { \$: Gonadenschutz während } \\
\text { Behandlung, } 0^{\top}: \text { Kryopreservation } \\
\text { von Spermien }\end{array}$ \\
\hline Leflunomid & Nicht bekannt & $\begin{array}{l}\text { Nicht bekannt ob mutagen, } \\
\text { daher auch bei } \sigma^{\top} \text { Auswaschen } \\
\text { empfohlen }\end{array}$ \\
\hline TNFa-Blocker & \$ nein, $\sigma^{\top}$ nicht geklärt & $\begin{array}{l}\text { Ob Beeinträchtigung der Sperma- } \\
\text { togenese, ist unzureichend unter- } \\
\text { sucht }\end{array}$ \\
\hline
\end{tabular}

Tab. 2 Antirheumatische Medikamente und Kombinationstherapien während der

Schwangerschaft

\begin{tabular}{lll} 
Medikament & $\begin{array}{l}\text { Absetzen vor der Schwanger- } \\
\text { schaft }\end{array}$ & $\begin{array}{l}\text { Maßnahmen während der } \\
\text { Schwangerschaft }\end{array}$ \\
NSAR & Nein & $\begin{array}{l}\text { Nichtselektive COX-Hemmer bis } \\
\text { Woche 32 erlaubt }\end{array}$ \\
$\begin{array}{l}\text { Sulfasalazin (SSZ) } \\
\text { Chloroquin und Hydroxy- } \\
\text { chloroquin (HChl) }\end{array}$ & Nein & $\begin{array}{l}\text { Folsäuresubstitution } \\
\text { Hydroxychloroquin ist vorzuziehen }\end{array}$ \\
\hline Cyclosporin & Nein & Dosis bei 2-3,5 mg/kg/Tag halten, \\
Azathioprin & Nein & Blutdruck der Mutter kontrollieren \\
Methotrexat (MTX) & 3 Monate vorher & Dosis bei 2 mg/kg/Tag halten \\
\hline Leflunomid & Absetzen und Auswaschen mit & Folsäuresubstitution beibehalten \\
Cholestyramin & Keine \\
\hline Mycophenolat mofetil & 6 Wochen vorher & Keine \\
\hline MTX, SSZ, HChl & MTX 3 Monate vorher & Folsäuresubstitution \\
\hline Prednison, MTX, SSZ & MTX 3 Monate vorher & Folsäuresubstitution \\
\hline TNFa-Blocker + MTX & MTX 3 Monate vorher & $\begin{array}{l}\text { TNFa-Blocker bei Eintritt der } \\
\text { Schwangerschaft absetzen }\end{array}$ \\
\hline
\end{tabular}

NSAR verzögerten Follikelsprungs zu erwägen und entsprechend zu untersuchen. Ein zeitweiliges Absetzen eines NSAR um die Zeit des Eisprungs kann Abhilfe schaffen.

\section{Medikamente und männliche Gonaden}

Oligo- oder Azoospermie kann durch Sulfasalazin und Cyclophosphamid verursacht werden. Die Beeinträchtigung der Spermatogenese unter Behandlung mit Methotrexat (MTX) ist selten und reversibel nach Abschluss der Therapie. Azathioprin und Cyclosporin schädigen die Spermatogenese nicht. Die durch Sulfasalazin ausgelösten Schäden der Spermatogenese sind etwa 2,5 Monate nach Absetzen der Therapie reversibel [19]. Die Behandlung mit Cyclophosphamid ist behaftet mit einem dosisabhängigen Risiko für eine irreversible Infertilität [7]. Leider kann man nicht voraussagen, welche Patienten steril werden oder ob und wann sich die Gonadenfunktion wieder erholt. Eine Rückkehr der Spermatogenese ist selbst nach 10 Jahren noch möglich (• Tab. 1).

\section{Amenorrhö unter Cyclophosphamid}

Die Behandlung mit Cyclophosphamid kann bei Frauen eine permanente Sterilität auslösen, jedoch ist diese vom Alter bei Beginn der Therapie und von der kumulativen Dosis abhängig [4]. Präpubertale Mädchen sind weniger gefährdet. Die Behandlung mit o,5-1,o g/ $\mathrm{m}^{2}$ Cyclophosphamid mehr als 10-mal erhöhte das Risiko für eine irreversible Infertilität bei Frauen $<25$ Jahren um etwa $12 \%$, bei Frauen im Alter zwischen 26-30 Jahren um etwa 27\% und stieg auf $62 \%$ an bei Frauen $>32$ Jahren [4]. Eine klar definierte kritische kumulative Dosis für irreversible Amenorrhö ist nicht bekannt. Auch weiß man nicht, ob Frauen, die wieder zu menstruieren beginnen, trotzdem eine verfrühte Menopause haben werden.

\section{Bewahrung der Gonadenfunktion}

Bei Mädchen nach eingetretener Pubertät ist die Behandlung mit einem Gonadotropin-releasing-hormone-Agonisten (GnRH-Agonisten) zu empfehlen [27]. Die Behandlung mit einem GnRH-Agonisten hindert effektiv die Follikelreifung und schützt das Ovar jedenfalls teilweise vor toxischer Wirkung der Zytostatika. Hormonelle Antikonzeption mit den heute meist niedrig dosierten Östrogenen hindert nicht komplett die Follikelreifung und scheint wenig geeignet, die Fertilität zu erhalten. Bisher liegen noch keine schlüssigen Daten vor, ob die gleichzeitige Gabe eines GonadotropinAgonisten eine Infertilität bei der Frau verhindern kann. Andere Methoden wie Kryopreservation von Ovar oder ovariellem Gewebe sind noch nicht hinreichend erprobt.

Bei Männern ist die beste Methode die Kryopreservation von Sperma vor dem Beginn einer Therapie mit Cyclophosphamid. Da die künstlichen Befruchtungsmethoden heute hoch entwickelt sind, ist dieses Vorgehen selbst bei reduzierter Anzahl oder verminderter Qualität von Spermien sinnvoll. 


\section{Basistherapie bei der Patientin mit Kinderwunsch}

Für alle rheumatischen Krankheiten gilt, dass eine Schwangerschaft möglichst in einer Phase der Remission und bei stabiler Medikation stattfinden sollte. Meist sind es Kombinationstherapien (• Tab. 2), die eine solche Remission aufrecht erhalten. MTX ist häufig Bestandteil der Therapie. Da es unbedingt vor Eintritt einer Schwangerschaft abgesetzt werden muss und andere Medikamente von Kombinationstherapien im Hinblick auf die Schwangerschaft z. T. unzureichend untersucht sind, ergeben sich Therapieprobleme. Bei einer Patientin mit Kinderwunsch eine bisher effektive Therapie abzusetzen, kann einen Schub provozieren, da es ungewiss ist, wie rasch die Patientin tatsächlich schwanger wird. Die Therapiemöglichkeiten für die Patientin mit Kinderwunsch werden im Folgenden dargestellt.

- Für Patientinnen mit RA, JIA oder Psoriasisarthropathie und Kinderwunsch ist Sulfasalazin als Monotherapie oder in Kombination mit einem Antimalarikum das Mittel der ersten Wahl.

Beide Medikamente können während der gesamten Schwangerschaft und Stillzeit eingenommen werden. In Deutschland wird die tägliche Verabreichung von Antimalarika während der Schwangerschaft und Stillzeit, im Gegensatz zu anderen Ländern, als Kontraindikation angesehen. Falls Sulfasalazin und Antimalarika wegen Unverträglichkeit oder Versagen der Behandlung nicht angewandt werden können, empfiehlt sich ein Versuch mit Azathioprin oder Cyclosporin (s. Abschn. „Therapie des SLE und anderer Kollagenosen“.

\section{Sulfasalazin}

Die Auswirkungen von Sulfasalazin auf den Verlauf und den Ausgang der Schwangerschaft wurde bei mehr als 2000 Graviditäten von Patientinnen mit entzündlichen Darmerkrankungen untersucht, die kontinuierlich oder vorübergehend Sulfasalazin allein oder in Kombination mit Kortikosteroiden erhielten. In keiner

Z Rheumatol 2006 -65:217-224 DOI 10.1007/s00393-006-0052-5

c) Springer Medizin Verlag 2006

\section{M. Østensen \\ Antirheumatische Therapie und Reproduktion. Einfluss auf Fertilität, Schwangerschaft und Stillzeit}

Zusammenfassung

Antirheumatische Medikamente können bei männlichen und weiblichen Patienten einen negativen Einfluss auf die Fortpflanzung haben. Mögliche negative Effekte sind: Beeinträchtigung der Fertiltät, Fruchtschädigung während der Schwangerschaft und Nebenwirkungen beim gestillten Kind. Bei der Frau können nichtsteroidale Antiphlogistika (NSAR) und Cyclophosphamid die Fertilität beeinträchtigen. Beim Mann kann Infertilität bei der Behandlung mit Salazopyrin und Cyclophosphamid auftreten.

Vor dem Start einer Therapie mit Basistherapeutika sollte abgeklärt sein, ob ein Kinderwunsch besteht. Die Behandlung mit NSAR ist in einigen Abschnitten der Schwangerschaft möglich, ebenso können sie während der Stillzeit eingesetzt werden. Eine begrenzte Anzahl von Basistherapeutika sind mit ei-

\section{Antirheumatic therapy and reproduction. the influence on fertility, pregnancy and breast feeding}

\section{Abstract}

Antirheumatic drugs can have a negative effect on reproduction in both men and women. Possible negative effects are impairment of fertility, harmful effects on the fetus and adverse effects on the breastfed child. In women non-steroidal antiinflammatory drugs (NSAID) and cyclophosphamide can impair fertility. In men infertility can result from the use of salazopyrine and cyclophosphamide. A desire for children should be taken into account before the start of disease modifying drugs (DMARD). Treatment with NSAID is possible at some stages of pregnancy as well as during lactation. A limited number of DMARD is compatible with pregnancy and is presented. Cytostatic drugs and leflu- ner Schwangerschaft vereinbar und werden vorgestellt. Medikamente wie Zytostatika und Leflunomid erfordern prophylaktisches Absetzen bereits vor einer geplanten Schwangerschaft. TNF a-Blocker müssen bei Eintritt einer Schwangerschaft abgesetzt werden. Bei Medikamenten, die toxisch für die Gonaden sind oder fruchtschädigend wirken können, muss während der Anwendung sicher verhütet werden. Die Anwendung von immunsuppressiven Medikamenten während der Stillzeit ist wegen unzureichender Datenlage sehr begrenzt.

Schlüsselwörter

Fertiltät · Schwangerschaft · Stillzeit · Nichtsteroidale Antiflogistika - Basismedikamente Cyclophosphamid·TNFa-Blocker nomide must be prophylactically withdrawn before a planned pregnancy. TNF alpha antagonists should be discontinued at the start of pregnancy. Safe birth control must be practised during therapy with drugs that are gonadotoxic or teratogenic. Treatment with immunosuppressive drugs during lactation is limited because of insufficient documentation of safety for the breastfed child.

Keywords

Fertility · Pregnancy $\cdot$ Breast feeding $\cdot$ Nonsteroid anti-inflammatory drugs $\cdot$ Disease modifying drugs . Cyclophosphamide . TNF alpha antagonists 
dieser Studien wurde eine Erhöhung der kindlichen Missbildungsrate gefunden oder ein vermehrtes Auftreten eines neonatalen Ikterus beobachtet $[17,18]$. Sulfasalazin ist ein Folsäureantagonist, sodass - obwohl kein erhöhtes teratogenes Risiko zu bestehen scheint - die Gabe eines Folsäurepräparates, z. B. $1 \mathrm{mg}$ täglich, für die gesamte Dauer der Schwangerschaft empfehlenswert ist [12].

\section{Antimalariamittel}

Die Verträglichkeit von Chloroquin und Hydroxychloroquin während der Schwangerschaft ist bei mehreren hundert Patientinnen mit SLE und RA untersucht worden $[3,9]$. Für die Standarddosierung $250 \mathrm{mg}$ Chloroquin oder 200$400 \mathrm{mg}$ Hydroxychloroquin wurden keine kindlichen Missbildungen berichtet. Auch Langzeituntersuchungen von Kindern, die in utero den Antimalarika ausgesetzt waren, zeigten keine Gesundheitsschäden an Auge oder Ohr [9].

Hydroxychloroquin hat eine geringere Verteilung als Chloroquin im Gewebe; auch wurde nie über kindliche Missbildungen berichtet. Für die Behandlung schwangerer Patientinnen ist es daher dem Chloroquin vorzuziehen.

\section{TNFa-Blocker}

Für die TNFa-Blocker Infliximab, Etanercept und Adalimumab sind bislang die Daten im Hinblick auf ihre Verträglichkeit während der Schwangerschaft unzureichend. Hinzu kommt, dass die beiden monoklonalen Antikörper Infliximab und Adalimumab eine Halbwertszeit von etwa 2 Wochen haben und eine vollständige Elimination individuell unterschiedlich mehrere Monate dauert. Aus diesem Grunde erscheint es ratsam, sie bei Eintritt einer Schwangerschaft abzusetzen.

Bei 40 Schwangerschaften, die sich während einer Behandlung mit Etanercept (Enbrel), und bei 131 Schwangerschaften, die sich während einer Infliximab-Therapie ereigneten, zeigte sich kein erhöhtes Risiko für Missbildungen oder andere Probleme des Neugeborenen [5, 14]. Über Adalimumab gibt es bisher nur 2 Fallberichte, beide mit unkompliziertem Ausgang der Schwangerschaft.
Prophylaktisch abzusetzende Medikamente

\section{Methotrexat (MTX)}

MTX ist ein Folsäureantagonist mit schädigender Wirkung auf den Fetus. Typische Missbildungen bei MTX-Behandlung im 1. Trimenon sind Neuralrohrdefekte sowie Schädel- und Skelettanomalien. Die kritische Phase für Missbildungen liegt zwischen der 6. und 8. Schwangerschaftswoche [11]. MTX kann auch eine Fehlgeburt auslösen.

In der rheumatologischen Literatur sind 63 Schwangerschaften mit einer versehentlichen Einnahme von MTX (in einer Dosierung von 7,5-20 mg/Woche) im 1. Trimenon beschrieben worden $[5,21]$. Zwei Kinder wiesen Missbildungen auf, die anderen Kinder waren gesund. Fasst man alle veröffentlichten Daten zusammen (auch die aus der onkologischen Literatur), muss bei Gabe von MTX im 1. Trimenon mit einem Missbildungsrisiko von etwa $10 \%$ gerechnet werden.

\section{- MTX darf grundsätzlich nur dann an fertile Frauen verordnet werden, wenn eine wirksame Empfängnisverhütung gewährleistet ist.}

Da aktive Metaboliten des MTX zwischen 2 und 3 Monaten in Körperzellen persistieren können, muss MTX unbedingt zumindest 3 Monate vor einer geplanten Schwangerschaft abgesetzt werden. Während der Behandlung mit MTX sollte ein Folsäurepräparat gegeben werden, das bei einer geplanten Schwangerschaft auch nach Absetzen des MTX weiterhin eingenommen und im 1 . Trimenon der Schwangerschaft beibehalten wird.

\section{Leflunomid}

Daten zur Einnahme von Leflunomid während der Schwangerschaft sind außerordentlich spärlich und lassen bisher keine Beurteilung zu, ob es tatsächlich eine für Menschen teratogene Substanz ist $[5,22]$. Da sich in Tierversuchen Missbildungen zeigten, muss von einer Gabe während der Schwangerschaft abgeraten werden. Da die Metaboliten des Leflunomid auch nach Absetzen noch etwa für
2 Jahre im Körper verbleiben, sollte bei Kinderwunsch mit Cholestyramin oder Aktivkohle ausgewaschen werden. Wenn nach zweimaliger Bestimmung des Plasmaspiegels kein Leflunomid mehr nachweisbar ist, kann eine Schwangerschaft durchgeführt werden.

\section{Ungeplante Schwangerschaft unter Therapie mit fetotoxischen Medikamenten}

Immer wieder kommt es vor, dass eine ungeplante Schwangerschaft unter der Behandlung mit Zytostatika oder potenziell fetotoxischen, immunsuppressiven Medikamenten eintritt. Um einen unnötigen und von der Patientin nicht gewollten Schwangerschaftsabbruch zu vermeiden, empfiehlt sich folgendes Vorgehen:

Der Zeitpunkt der Medikamenteneinnahme sollte genau erhoben werden. Falls die Einnahme in den ersten 2 Wochen der Schwangerschaft erfolgte, ist kein Schaden zu befürchten, denn für diese Phase gilt das Alles-oder-Nichts-Gesetz. Eine toxische Substanz vernichtet entweder die befruchtete Eizelle oder es findet eine komplette Erholung nach einer partiellen Schädigung statt. Bei späterer Exposition sollte die für das Medikament kritische Phase einer Fruchtschädigung bekannt sein. Außerhalb dieses Zeitfensters ist eine Fruchtschädigung nicht zu erwarten.

Weitere Maßnahmen sind eine Chorionzottenbiopsie zum Ausschluss von Chromosomenstörungen und die Ultraschalluntersuchung des Fetus zwischen der 11. und 12. Schwangerschaftswoche und erneut um die 17. Woche zum Ausschluss von Organmissbildungen. Falls alle diese Untersuchungen einen normalen Fetus zeigen, kann von einer ernsthaften Fruchtschädigung abgesehen werden.

\section{Die Behandlung einer akuten oder persitierend aktiven Arthritis während der Schwangerschaft}

\section{Nichtsteroidale Antirheumatika}

Bei der Mehrzahl der Patientinnen mit Spondyloarthropathien und bei etwa $25 \%$ mit einer Polyarthritis bleibt die Arthritis während der Schwangerschaft aktiv oder verschlechtert sich akut. Oft sind 


\section{Hier steht eine Anzeige.}

刑 Springer 
die Symptome im 1. und 2. Drittel der Schwangerschaft am stärksten ausgeprägt und erfordern eine Therapie mit NSAR.

Die nichtselektiven Zyklooxygenasehemmer wie Salicylate, Indomethacin, Fenoprofen, Ibuprofen, Naproxen, Diclofenac, Mefenaminsäure und Piroxicam sind nicht teratogen, wie mehrere epidemiologische Studien gezeigt haben. Sie brauchen daher weder vor einer Schwangerschaft noch im 1. Trimenon abgesetzt zu werden [22].

Über die selektiven Cox-2-Hemmer gibt es bisher nur spärliche Daten, sodass es ratsam erscheint, sie bei Eintritt einer Schwangerschaft abzusetzen und auf ein klassisches NSAR zu wechseln.

\section{- Im letzten Trimenon der Schwangerschaft kann sowohl eine selektive als auch eine nichtselektive Hemmung der Zyklooxygenasen 1 und 2 (COX 1 , COX 2) bedeutsame Nebenwirkungen beim Feten verursachen.}

Infolge der Hemmung der fetalen Prostaglandinsynthese wird die renale Durchblutung vermindert [13]. Daraus resultieren eine Reduktion der fetalen Urinproduktion und ein vermindertes Volumen der Amnionflüssigkeit. Falls NSAR bis kurz vor der Entbindung gegeben werden, kann auch die Nierenfunktion des Neugeborenen beeinträchtigt sein. Die renalen Nebenwirkungen beim Kind sind nach Absetzen des NSAR in der Regel innerhalb von 1-4 Tagen reversibel [22].

Klinische und experimentelle Studien haben gezeigt, dass alle COX-Hemmer den Ductus Botalli verengen können [22]. Der konstringierende Effekt der NSAR auf den Ductus arteriosus wurde ab der 27. Schwangerschaftswoche nachgewiesen [29]. Er ist gewöhnlich innerhalb von 2472 Stunden nach Absetzen des NSAR reversibel, wie echokardiographische Untersuchungen gezeigt haben. Bei Neugeborenen, deren Mütter in der späten Schwangerschaft NSAR eingenommen hatten, zeigte sich häufiger eine pulmonale $\mathrm{Hy}$ pertonie [1]. Generell sind die genannten Nebenwirkungen vermehrt bei prämaturen Neugeborenen zu beobachten, die noch kurz vor der Geburt den Wirkungen eines NSAR ausgesetzt waren.
Im 1. und 2. Trimenon können nichtselektive NSAR gegeben werden, in der geringsten effektiven Dosis und begrenzt auf aktive Krankheitsphasen. Falls die Intensität der Beschwerden von der Tageszeit abhängt, kann man die Dosierung eines NSAR mit kurzer oder mittlerer Halbwertszeit entsprechend anpassen. Nächtliche Gelenkschmerzen und ausgeprägte Morgensteifigkeit können durch die abendliche Gabe evtl. mit doppelter Dosierung des NSAR gemildert werden. Eine NSAR-Behandlung während des Tages kann dann überflüssig sein.

\section{() NSAR 6-8 Wochen vor der Geburt absetzen}

Um Nebenwirkungen auf die Niere oder den Ductus arteriosus beim Feten oder Neugeborenen zu vermeiden, müssen NSAR 6-8 Wochen vor der Geburt abgesetzt werden. Falls dies nicht möglich ist, z. B. bei unbefriedigender Wirkung von Paracetamol oder hochdosiert angewandten Kortikosteroiden, kann ein NSAR mit kurzer Halbwertszeit unter sonographischer Überwachung des Fetus gegeben werden. Sollten sich dabei aber kardiale oder renale Nebenwirkungen zeigen, muss das NSAR sofort abgesetzt werden.

Ein Aufflammen einer Entzündung in peripheren Gelenken kann mit Kortikosteroidinjektionen ins Gelenk behandelt werden. Prednison oder Prednisolon in einer Dosierung von 5-10 mg täglich oder eine ein- oder mehrmalige intramuskuläre Injektion von 40-80 mg Methylprednisolon sind ebenfalls Therapie der Wahl, wenn bei Patientinnen mit aktiver Gelenkentzündung das NSAR während der letzten Schwangerschaftswochen abgesetzt wird.

Gelenkschmerzen können in allen Abschnitten der Schwangerschaft mit Paracetamol in der üblichen Dosierung 1-4 g täglich behandelt werden.

\section{Therapie des SLE und anderer Kollagenosen}

Die Behandlung einer aktiven Arthritis bei einer schwangeren SLE-Patientin unterscheidet sich nicht von dem oben angegeben Vorgehen. Organkomplikationen können dagegen schwerwiegend sein und eine potente Therapie erfordern. Die Kombination von Kortikosteroiden und immunsuppressiven Medikamenten ist dann angezeigt (• Tab. 2).

\section{Kortikosteroide}

Die verschiedenen Kortikosteroide gelangen in unterschiedlichem Maße durch die Plazentaschranke in den Blutkreislauf des Fetus. Prednison wird in der Leber in das aktive Prednisolon umgewandelt. Prednisolon wird weitgehend von den 11Betahydroxylasen der Plazenta inaktiviert. Aus diesem Grund sind Prednisolon und Prednison zur Behandlung schwangerer Frauen in allen Abschnitten der Schwangerschaft geeignet, doch sollte die Tagesdosis im 1. Trimenon $15 \mathrm{mg}$ nicht überschreiten, da sonst ein leicht erhöhtes Risiko für das Auftreten einer Lippen-Gaumen-Spalte besteht $[10,23]$.

\section{- Eine Prednison-Behandlung der Mutter mit Dosen $>20$ mg/Tag in späteren Abschnitten der Schwangerschaft erhöht das Risiko für prämature Geburt und für ein reduziertes Geburtsgewicht des Kindes.}

Bei sehr aktiver, systemischer Krankheit oder bei Organkomplikationen kann man die Schwangere kurzfristig mit 1-2 mg/kg Prednison oder Prednisolon täglich behandeln.

\section{Azathioprin}

Azathioprin ist als Bestandteil der immunsuppressiven Behandlung bei Transplantierten im Hinblick auf die Verträglichkeit während der Schwangerschaft sehr gut untersucht. Wenn notwendig, kann es auch während des 1. Trimenons eingenommen werden. Dem Feten mangelt das Enzym, das Azathioprin in seinen aktiven Metaboliten umwandelt, und schützt ihn daher vor seinem Effekt auf die Zellteilung. Analysen von Schwangerschaften bei transplantierten Patienten unter immunsuppressiver Therapie haben kein erhöhtes Risiko für angeborene Anomalien gefunden [25]. Zum gleichen Resultat kamen Studien bei schwangeren SLE-Patientinnen [26]. Um einen negativen Ef- 
Tab. 3 Antirheumatische Medikamente und Stillen

\begin{tabular}{|c|c|c|}
\hline Medikament & Übergang in die Muttermilch & Beim Stillen zulässig \\
\hline NSAR & In geringen Mengen & $\begin{array}{l}\text { Diclofenac, Flufenaminsäure, Ibu- } \\
\text { profen, Indomethacin, Ketorolac, } \\
\text { Mefenaminsäure, Naproxen, Piroxi- } \\
\text { cam sind erlaubt }\end{array}$ \\
\hline Prednison & In geringen Mengen & $\mathrm{Ja}$ \\
\hline Sulfasalazin & $\mathrm{Ja}$ & Ja \\
\hline $\begin{array}{l}\text { Chloroquin und Hydroxy- } \\
\text { chloroquin }\end{array}$ & In geringen Mengen & $\mathrm{Ja}$ \\
\hline Cyclosporin & $\begin{array}{l}\text { Sekretion in die Milch sehr } \\
\text { unterschiedlich }\end{array}$ & Nein \\
\hline Azathioprin & $\mathrm{Ja}$ & Nein \\
\hline Methotrexat (MTX) & In geringer Menge & $\begin{array}{l}\text { Nein - Wirkung auf das gestillte Kind } \\
\text { unbekannt }\end{array}$ \\
\hline Cyclophosphamid & $\mathrm{Ja}$ & Nein \\
\hline Leflunomid & Nicht untersucht & Nein \\
\hline Mycophenolat mofetil & Nicht untersucht & Nein \\
\hline Infliximab & Geringe Mengen & $\begin{array}{l}\text { Nein - Wirkung auf das gestillte Kind } \\
\text { unbekannt }\end{array}$ \\
\hline Etanercept & Geringe Mengen & $\begin{array}{l}\text { Nein - Wirkung auf das gestillte Kind } \\
\text { unbekannt }\end{array}$ \\
\hline Adalimumab & Nicht untersucht & Nein \\
\hline
\end{tabular}

fekt auf die Hämatopoese des Neugeborenen zu vermeiden, sollte die Dosis bei einer Schwangeren $2 \mathrm{mg} / \mathrm{kg} /$ Tag nicht überschreiten.

\section{Cyclosporin}

Es sind mehr als 800 Schwangerschaften unter der Einnahme von Cyclosporin publiziert. Eine Metaanalyse von 410 Schwangerschaften bei Patientinnen mit Allotransplantaten oder autoimmunen Krankheiten fand keine Erhöhung der kindlichen Missbildungsrate verglichen mit unbehandelten Kontrollen [2]. Nachuntersuchungen von Kindern, deren Mütter während der Schwangerschaft mit Cyclosporin behandelt wurden, zeigten keine Entwicklung von Autoimmunität oder Immundefizienz [6]. Wenn eine Therapie mit Cyclosporin indiziert ist, kann es während der Schwangerschaft eingenommen werden. Die Dosis sollte $2-3,5 \mathrm{mg} / \mathrm{kg} /$ Tag nicht überschreiten. Bei höherer Dosis besteht ein Risiko für eine intrauterine Wachstumsstörung.

\section{Mycophenolat mofetil (CellCept) und Tacrolimus}

CellCept und Tacrolimus werden zunehmend für die Behandlung von Or- gankomplikationen des SLE und anderer Kollagenosen verwendet. Die bisher spärlichen Daten zu Schwangerschaften, die sich unter Einnahme von CellCept ereigneten, schließen die Möglichkeit einer teratogenen Wirkung nicht aus [22, 24]. Daher sollte CellCept nur bei gewährleisteter sicherer Verhütung an fertile Frauen verschrieben werden. Wegen seiner langen Halbwertszeit sollte CellCept 6 Wochen vor einer geplanten Schwangerschaft abgesetzt werden.

Die bisher veröffentlichten Erfahrungen mit Tacrolimus lassen keine schädigende Wirkung auf die humane Schwangerschaft erkennen [24]. Bei strenger Indikation scheint daher die Weiterbehandlung mit Tacrolimus auch während einer Schwangerschaft möglich.

\section{Cyclophosphamid}

Cyclophosphamid ist teratogen, auch wenn eine Verabreichung im 1. Trimenon nicht immer zu kindlichen Missbildungen führen muss [16].

\section{- Eine Behandlung nach dem}

1. Trimenon kann zu intrauteriner

Wachstumsstörung, Störung der neurologischen Entwicklung und der Hämatopoese des Kindes führen.
Die bei einer Lupusnephritis häufig durchgeführte intravenöse Behandlung mit Cyclophosphamid sollte nur nach Vorliegen eines negativen Schwangerschaftstests durchgeführt werden. Sollte es während einer Schwangerschaft zu einer aktiven Lupusnephritis kommen, ist nach dem 1. Trimenon die Behandlung mit Cyclophosphamid in schweren Fällen möglich.

\section{Antirheumatika und Stillen}

In der Literatur gibt es nur sehr wenige Mitteilungen über die Ausscheidung von Antirheumatika über die Muttermilch. Die meisten Substanzen werden nur in sehr geringer Menge mit der Milch ausgeschieden (• Tab. 3). Die Ergebnisse der veröffentlichten Untersuchungen:

Chloroquin, Hydroxychloroquin und Sulfasalazin [8] können unbedenklich von der stillenden Patientin eingenommen werden. Infliximab und Etanercept gehen in die Muttermilch über. Mögliche Wirkungen dieser Medikamente auf den Säugling sind nicht untersucht, und die Behandlung während der Stillzeit sollte daher vermieden werden. Cyclosporin geht in unterschiedlichen Mengen in die Muttermilch über. Bei 14 Kindern, die von mit Cyclosporin behandelten Müttern gestillt wurden, traten keine Nebenwirkungen auf. Dennoch wird aus theoretischen Erwägungen vom Stillen abgeraten. Über die Ausscheidung von Leflunomid in die Muttermilch liegen keine Informationen vor.

Nur sehr geringe Mengen von Prednison und Prednisolon treten in die Milch über, weshalb das Stillen auch bei langfristiger Behandlung erlaubt werden kann [8]. Selbst bei einer Tagesdosis von $80 \mathrm{mg}$ ist die errechnete Menge Prednison, die der Säugling beim Stillen zu sich nimmt, geringer als 10\% seiner endogenen Kortisolproduktion.

Die Ausscheidung von Azathioprin, Cyclophosphamid und MTX in die Muttermilch ist erwiesen [8]. Neun Kinder, die durch die Brustmilch dem Azathioprin ausgesetzt waren, wiesen keine $\mathrm{Ne}$ benwirkungen auf. Über Zytopenie bei einem Säugling, der von einer mit Cyclophosphamid behandelten Mutter gestillt wurde, ist berichtet worden. Mitteilungen 
über Nebenwirkungen von MTX bei dem mit Brustmilch ernährten Säugling fehlen. Da man grundsätzlich mit einer immunosuppressiven und zytotoxischen Wirkung von Zytostatika rechnen muss, sollten sie nicht an Stillende verabreicht werden.

Da die meisten NSAID schwache Säuren sind, werden sie in sehr geringer Menge in der Muttermilch ausgeschieden [28]. Wie - Tab. 3 zeigt, können mehrere NSAID unbedenklich während des Stillens eingenommen werden. Ibuprofen ist das Mittel der ersten Wahl, da es nicht (oder jedenfalls nicht in messbaren Mengen) mit der Milch ausgeschieden wird.

\section{- Eine längere Behandlung mit 1-3 g/Tag eines Acetylsalicyl- säurederivates sollte vermieden werden, da die Ausscheidung in die Milch stark variiert und daher teils beträchtliche Konzentrationen in der Milch gefunden werden.}

Eine Patientin, die mit einem oder mehreren Antirheumatika behandelt wird, sollte die Medikamente unmittelbar vor oder nach dem Stillen einnehmen. Es wird außerdem empfohlen, das Kind erst wieder nach so vielen Stunden anzulegen, wie die Halbwertszeit des einzelnen Medikaments beträgt. Aus dieser Überlegung heraus sind Medikamente mit kurzer Halbwertszeit vorzuziehen.

\section{Fazit für die Praxis}

Eine medikamentöse Therapie während der Schwangerschaft und Stillzeit ist möglich, wenn auch die Anzahl der zulässigen Medikamente wegen der oft unzureichenden Datenlage begrenzt ist. Entzündliche Schmerzen, ausgelöst durch eine aktive Arthritis oder Spondylitis, sollten effektiv behandelt werden. Tritt eine Besserung ein, können die Antirheumatika entweder abgesetzt oder in der Dosis reduziert werden. Bei Kollagenosen mit Organbeteiligung sollte eine immunsuppressive Therapie auch während der Schwangerschaft und Stillzeit beibehalten werden, um einen Schub zu verhindern. Um die Aktivität der Krankheit zu bremsen, ohne den Fetus oder das Neugeborene zu beeinträchtigen, muss die Strategie lauten: So wenig Me- dikamente wie möglich, aber so viel wie nötig.

\section{Korrespondierender Autor}

\section{Prof. Dr. M. Østensen}

Klinik für Rheumatologie und Klinische Immunologie/Allergologie, Inselspital Bern, Universitätsklinik

3010 Bern

monika.oestensen@insel.ch

Interessenkonflikt. Es besteht kein Interessenkonflikt. Der korrespondierende Autor versichert, dass keine Verbindungen mit einer Firma, deren Produkt in dem Artikel genannt ist, oder einer Firma, die ein Konkurrenzprodukt vertreibt, bestehen. Die Präsentation des Themas ist unabhängig und die Darstellung der Inhalte produktneutral.

\section{Literatur}

1. Alano MA, Ngougmna E, Ostrea EM Jr, Konduri GG (2001) Analysis of nonsteroidal antiinflammatory drugs in meconium and its relation to persistent pulmonary hypertension of the newborn. Pediatrics 107: 519-513

2. Bar-Oz B, Hackman R, Einarson T, Koren G (2001) Pregnancy outcome after cyclosporine therapy during pregnancy: a meta-analysis. Transplantation 71: 1051-1055

3. Borden MB, Parke AL (2001) Antimalarial drugs in systemic lupus erythematosus. Use in pregnancy. Drug safety 24: 1055-1063

4. Boumpas DT, Howard AA, Vaughan EM et al. (1993) Risk of sustained amenorrhea in patients with systemic lupus erythematosus receiving intermittent pulse cylcophosphamide therapy. Ann Intern Med 119: 366-369

5. Chakravarty EF, Sanchez-Yamamoto D, Bush TM (2003) The use of disease modifying antirheumatic drugs in women with rheumatoid arthritis of childbearing age: a survey of practice patterns and pregnancy outcome. J Rheumatol 30: 241-246

6. Cimaz R, Meregalli E, Biggioggero M et al. (2004) Alterations in the immune system of children from mothers treated with immunosuppressive agents during pregnancy. Toxicol Lett 149: 155-162

7. Clovis A, Silva A, Hallak J et al. (2002) Gonadal function in male adolescents and young males with juvenile onset systemic lupus erythematosus. J Rheumatol 29: 2000-2005

8. Committee on Drugs. American Academy of pediatrics (2001) The transfer of drugs and other chemicals into human milk. Pediatrics 108: 776-789

9. Costedoat-Chalumeau N, Amoura Z et al. (2003) Safety of hydroxychloroquine in pregnant patients with connective tissue diseases: a study of one hundred thirty-three cases compared with a control group. Arthritis Rheum 48: 3207-3211

10. Czeizel AE, Rockenbauer M (1997) Population-based case-control study of teratogenic potential of corticosteroids. Teratology 56: 335-340

11. Feldkamp M, Carey JC (1993) Clinical teratology counseling and consultation case report: low dose methotrexate exposure in early weeks of pregnancy. Teratology 47: 533-539

12. Hernandez-Diaz S, Werler MM, Walker AM, Mitchel AA (2000) Folic acid antagonists during pregnancy and the risk of birth defects. N Eng J Med 343: 1608-1614
13. Hickok DE, Hollenbach KA, Reilley SF, Nyberg DA (1989) The association between decreased amniotic fluid volume and treatment with nonsteroidal anti-inflammatory agents for preterm labor. Am J Obstet Gynecol 160: 1525-1531

14. Katz JA, Antoni C, Keenan GF et al. (2004) Outcome of pregnancy in women receiving Infliximab for the treatment of Crohn's disease and rheumatoid arthritis. Am J Gastroenterol 99: 2385-2392

15. Mendonca LLF, Khamashta MA, Nelson-Piercy C, Hughes GRV (2000) Non-steroidal anti-inflammatory drugs as a possible cause for reversible infertility. Rheumatology 39: 880-882

16. Mirkes PE (1985) Cyclophosphamide teratogenesis. A review. Teratogen Carcinogen Mutagen 5: 75-88

17. Mogadam M, Dobbins WO, Korelitz BI, Ahmed SW (1981) Pregnancy in inflammatory bowel disease: effect of sulphasalazine and corticosteroids on fetal outcome. Gastroenterology 80: 72-76

18. Norgard B, Czeizel AE, Rockenbauer M et al. (2001) Population based case control study of the safety of sulfasalazine use during pregnancy. Aliment Pharmacol Ther 15: 483-486

19. O'Morain C, Smethurst P, Dore CJ et al. (1984) Reversible male infertility due to sulphasalazine: studies in man and rat. Gut 25: 1078-1084

20. Østensen M, Østensen H (1996) Safety of nonsteroidal antiinflammatory drugs in pregnant patients with rheumatic disease. J Rheumatol 23: 1045-1049

21. Østensen M, Hartmann H, Salvesen K (2000) Low dose weekly methotrexate in early pregnancy. A case series and review of the literature. J Rheumatol 27: 1872-1875

22. Østensen $M$, Khamashta M, Lockshin M et al. (2006) Antirheumatic drugs and reproduction. Arthritis Res Ther (in press)

23. Park-Wyllie L, Mazzotta P, Pastuszak A et al. (2000) Birth defects after maternal exposure to corticosteroids: Prospective cohort study and meta-analysis of epidemiological studies. Teratology 62: 385392

24. Pergola PE, Kancharla A, Riley DJ (2001) Kidney transplantation during the first trimenon of pregnancy: immunosuppression with mycophenolate mofetil, tacrolimus, and prednisone. Transplantation 71: 994-997

25. Polifka JE, Friedman JM (2002) Teratogen update: Azathioprine and 6-Mercaptopurine. Teratology 65: 240-261

26. Ramsey-Goldman R, Mientus JM, Kutzer JE et al. (1993) Pregnancy outcome in women with systemic lupus erythematosus treated with immunosuppressive drugs. J Rheumatol 20: 1152-1157

27. Slater CA, Liang MH, McCune JW et al. (1999) Preserving ovarian function in patients receiving cyclophosphamide. Lupus 8: 3-10

28. Spigset O, Hägg S (2000) Analgesics and breastfeeding. Safety considerations. Paediatric Drugs 2: 223-238

29. Vermillion ST, Scardo JA, Lashus AG, Wiles HB (1997) The effect of indomethacin tocolysis on fetal ductus arteriosus constriction with advancing gestational age. Am J Obstet Gynecol 177: 256261 